\title{
Théologiques
}

\section{La conversion}

\section{Le tournant monastique $\mathrm{dlu}_{\mathrm{IV}}^{\mathrm{e}}$ siècle}

\section{Fabrizio Vecoli}

Volume 21, numéro 2, 2013

La conversion

URI : https://id.erudit.org/iderudit/1028461ar

DOI : https://doi.org/10.7202/1028461ar

Aller au sommaire du numéro

\section{Éditeur(s)}

Faculté de théologie et de sciences des religions, Université de Montréal

ISSN

1188-7109 (imprimé)

1492-1413 (numérique)

Découvrir la revue

Citer cet article

Vecoli, F. (2013). La conversion : le tournant monastique du IV ${ }^{\mathrm{e}}$ siècle. Théologiques, 21(2), 17-41. https://doi.org/10.7202/1028461ar
Résumé de l'article

La conversion est au départ un phénomène judéo-chrétien, donc culturellement très connoté. Et c'est précisément dans cette perspective limitée que se situe notre intervention. L'intérêt est celui de mieux comprendre d'un point de vue historique - une phase clé de l'évolution de la notion de conversion dans l'histoire du christianisme ancien. Il s'agit ici de remonter aux éléments premiers qui ont forgé le sens du mot tel que nous l'utilisons aujourd'hui en Occident. On ne se propose pas de forger la définition ultime, correspondante à la chose même à sa naissance, mais plutôt de saisir la dimension évolutive - donc dynamique - de la conversion, à travers l'étude d'une période charnière de son développement : c'est ce que l'on nomme ici le tournant monastique (IV- $\mathrm{V}^{\mathrm{e}}$ siècles). Nous voulons montrer comment le phénomène de la conversion s'est transformé de façon significative à partir du $\mathrm{IV}^{\mathrm{e}}$ siècle de notre ère.
Ce document est protégé par la loi sur le droit d'auteur. L'utilisation des services d’Érudit (y compris la reproduction) est assujettie à sa politique d'utilisation que vous pouvez consulter en ligne.

https://apropos.erudit.org/fr/usagers/politique-dutilisation/ 


\title{
La conversion
}

\section{Le tournant monastique du IV $^{\mathrm{e}}$ siècle}

\author{
Fabrizio VeCOLI* \\ Histoire du christianisme \\ Université de Montréal (Canada)
}

\section{La conversion dans le christianisme ancien}

Le phénomène de la conversion est un objet d'étude complexe et un sujet d'analyse controversé (Rambo-Farhadian 2005). Dans les sciences religieuses, les approches méthodologiques susceptibles d'être déployées pour appréhender cette thématique sont de toute évidence très diversifiées, mais la véritable difficulté semble venir de la nature polémique intrinsèque - et difficile à désamorcer - de la conversion. Pour s'en rendre compte, il peut être utile de lire, en référence à la période de l'Antiquité tardive qui nous intéresse ici, la Lettre sur la conversion des juifs de l'évêque Sévère de Minorque, diffusée en 418, soit après l'entrée dans la foi chrétienne des juifs de l'île (Bradbury 1996). Un saut jusqu'à l'entrée "conversion », dans un dictionnaire théologique récent (Goffi 2001, 198), et l'on constatera que l'explication de la notion glisse facilement — encore aujourd'hui — dans la controverse ${ }^{1}$ : la conversion y est présentée comme une étape nécessaire dans le «processus de maturation " auquel l'être humain est appelé, en tant qu'individu et en tant qu'espèce. De là à l'identifier avec la "gratia

* Fabrizio Vecoli est professeur adjoint à la Faculté de théologie et de sciences des religions de l'Université de Montréal. Son travail de recherche se concentre actuellement sur le concept de discernement dans l'histoire du christianisme. Parmi ses œuvres, la monographie (2007) Il sole e il fango. Puro e impuro tra i Padri del deserto, Rome, Edizioni di Storia e Letteratura reflète son intérêt pour l'histoire du monachisme ancien.

1. «[L’Église] ne renonce cependant pas à faire croître les valeurs qui existent dans les autres religions, puisqu'elle n'ignore pas que c'est seulement chez elle, dans l'Esprit du Christ, que "les hommes trouvent la plénitude de la vie religieuse et la réconciliation de toutes choses en Dieu” " (p. 198).

(C) Revue Théologiques 2013. Tout droit réservé. 
gratum faciens » d'Augustin², il s'en faut de peu (Lonergan 1972, 34; 1972, 240-241). Dans une perspective plus impartiale, un nombre important de chercheurs s'accordent pour définir la conversion comme un religious change. Il s'agit d'une locution quelque peu vague qui, hélas, n'évite pas - quant au déchiffrement de la notion désignée — l'écueil d'une dépendance directe de la position adoptée envers le phénomène religieux en général. En ce sens, "conversion» est fonction de «religion». Un point de vue «interne» en saisira certes la nature authentiquement religieuse, mais risquera également de tomber dans le jugement de valeur; un point de vue "externe», d'autre part, permettra sans doute une certaine impartialité épistémologique, mais le spectre du réductionnisme hantera toute analyse. La navigation entre ces Scylla et Charybde de l'étude du religieux s'avère dès lors particulièrement périlleuse.

Cela dit, si l'on excepte la notion d' «illumination» dans le bouddhisme, la conversion est au départ un phénomène judéo-chrétien, donc culturellement très connoté. Et c'est précisément dans cette perspective limitée du "culturellement connoté » que se situe notre intervention, dans le sens où l'intérêt sera celui de mieux comprendre - d'un point de vue historique - une phase clé de l'évolution de la notion de conversion dans l'histoire du christianisme ancien. En effet, il n'est jamais inutile de revenir à l'Antiquité, car, malgré tout, c'est bien de là que nous venons (Borgeaud $2004,17^{3}$ ). Malgré la légitimité de toute approche structuraliste ou cognitive, le but ici sera plutôt de remonter aux éléments premiers qui ont forgé le sens du mot conversion tel que nous l'utilisons aujourd'hui en Occident. Du moins voudra-t-on en préciser certaines nuances. Non que l'on veuille affirmer l'existence d'une essence première de cette réalité, éventuellement atteignable par le biais d'une archéologie sémantique des origines. Le but n'est évidemment pas de forger la définition ultime, correspondante à la chose même à sa naissance, mais plutôt de saisir la dimension évolutive, donc dynamique, de la conversion, à travers l'étude d'une période charnière

2. Sans l'intervention divine, la sanctification de l'homme reste inaccessible: cela reprend la notion de conversion comme appel au salut.

3. «La véritable matière de l'histoire des religions, celle qu'il est devenu de plus en plus urgent d'étudier, ce n'est pas la série des grandes ou petites religions du monde, mais bien les éléments anciens et les mécanismes souvent archaïques, et résistants, à partir desquels les croyances actuelles ont été élaborées [...]. Il faut comprendre que ce dépôt, ce serait un peu comme l'inconscient de la civilisation humaine plus ou moins refoulé». 
de son développement: c'est ce que l'on nommera le tournant monastique (IV-V siècles).

Notre but est donc de montrer comment le phénomène de la conversion s'est transformé de façon significative à partir du IV siècle de notre ère, soit - pour emprunter les mots de l'historien français Paul Veyne — «quand notre monde est devenu chrétien» (Veyne 2007). Or, cette transformation apparaît tout à fait pertinente pour appréhender l'acception de la notion de conversion telle que comprise et vécue à l'époque contemporaine.

\section{Le terme}

Si l'on traite la conversion comme un concept (qui découle assurément d'une pratique), la nécessité d'une brève analyse terminologique paraitra évidente, car l'étymologie du terme évoque l'histoire de son utilisation. On sait effectivement que "conversion» vient du latin conversio, qui indique l'acte de se retourner (verbe convertire). Toutefois, il faut remonter au grec, langue commune des premiers disciples de Jésus, pour saisir toute la richesse du concept dans le christianisme ancien. C'est dans ce contexte qu'il a pris son essor, devenant ainsi une notion fondamentale de la vie religieuse méditerranéenne. Or, le grec a, pour ce qu'on nomme aujourd'hui conversion, deux termes: epistrophē, qui est l'équivalent du conversio latin dans la signification de «torsion, retournement », et metanoia, qui indique un changement d'opinion, mais aussi le regret et la pénitence pour les péchés commis (Lampe 1961, 536 et 855). Il y a donc, à la base, dans le lexique ancien de la conversion, un jugement de valeur, à la fois négatif sur le passé - un passé qui doit être rejeté - et positif sur le présent du converti, qui a finalement accès à la vraie voie de salut (Moloney, Lawrence et Kirwin 2003, 232). Bref, le terme grec de metanoia introduit une dimension pénitentielle dans l'idée de conversion que l'on retrouvera dans le monachisme (Miquel 1986, 181-190).

D'ailleurs, metanoia et epistrophē sont associés dès les débuts de la prédication de Jésus, car l'appel au changement et au renouveau lancé par le Christ s'exprime justement par ces deux termes ou, plutôt, par les verbes correspondants. En effet, dans Matthieu 18,3, il est dit: «En vérité je vous le dis, si vous ne vous retournez (straphete) et ne devenez pas comme cet enfant, vous n'entrerez pas dans le Royaume des cieux. » Dans Marc 1,15, on précise les conditions requises (repentance et foi) de ce "retournement» : «Le temps est accompli et le Royaume de Dieu est proche: repentez-vous (metanœite) et croyez à l'Évangile.» 
Quoi qu'il en soit de la complexe histoire rédactionnelle de ces textes, les auteurs chrétiens successifs vont s'y référer ${ }^{4}$. Indépendamment de la pratique réelle - qui certes s'avèrera souvent contradictoire - la conversion sera idéalement considérée comme un changement de direction, impliquant une contrition à l'égard du passé. D'ailleurs, on y associera souvent le thème des deux voies (Moreschini-Norelli 2000, 162, Attridge 2000, 204), soit la nécessité du choix que tout croyant doit faire entre la lumière (ou la vie) et les ténèbres (ou la mort). C'est là un thème récurrent des premiers écrits chrétiens (Didaché 1,1-6,3; Doctrine des Apôtres 4-14, Constitution des Apôtres 1,1 ss.), qui l'ont certainement reçu du judaïsme ancien (à Qumran: Règle de la communauté 3,13-4,26). Mais ce thème se retrouve aussi bien dans la philosophie grecque (Xénophon, Memorabilia II,1,18-34) et remonte vraisemblablement au zoroastrisme ancien (Duhaime 2000, 219) $)^{5}$, considéré comme la première tradition à proposer au fidèle le thème du choix entre le bien et le mal. Or, cette dichotomie est très clairement associée à la conversion dans l'Épitre du Pseudo-Barnabé $(11,8)$, où le terme epistrophē se réfère au choix du chrétien qui s'engage sur la voie du salut, opposée à celle qui mène les impies à la damnation. La différence par rapport au thème classique des deux voies réside dans le fait que, dans la théorie chrétienne, on présuppose que le mauvais choix a déjà été accompli : il faut donc le corriger. On comprendra alors qu'Augustin d'Hippone (354-430) ${ }^{6}$ ait pu expliquer l'étymologie du mot latin religio en le faisant dériver du verbe re-eligĕre, "re-choisir » : le terme désignerait un retour sur le bon chemin à la suite d'un regrettable égarement. Conversion et religion seraient donc soudées dans le même mot, signe représentatif d'une nouvelle conception qui s'impose avec le christianisme de l'Antiquité tardive (Filoramo 2004, 82). La dimension polémique de la notion de conversion, ici évidente, s'était manifestée de façon claire dès le $\mathrm{II}^{\mathrm{e}}$ siècle, dans le Dialogue avec Tryphon $(30,1)$ de Justin († vers 165): le terme

4. Pour se rendre compte de l'importance de ces deux passages évangéliques dans la patristique, il suffira de lancer une recherche sur Biblindex, l'équivalent online de la Biblia Patristica: <http://www.biblindex.mom.fr/>.

5. Je tiens à remercier le professeur et collègue Jean Duhaime pour m'avoir signalé son article, ainsi que celui de Attridge cité plus haut.

6. «Hunc [Deum] eligentes vel potius religentes, amiseramus enim negligentes, bunc ergo religentes unde religio dicta perhibetur, ad Eum dilectatione tendimus ut perveniendo quiescamus (en le choisissant ou plutôt en le re-choisissant - nous l'avions égaré par négligence - donc en le re-choisissant, d'où vient - à ce qu'on dit — le mot religion, nous tendons à lui par l'amour afin que, l'ayant rejoint, nous trouvions le repos)»(De Civitate Dei $\mathrm{X}, 4)$. 
metanoia y était utilisé dans le cadre de la controverse entre chrétiens et juifs.

Il est donc légitime d'affirmer que l'appel à la conversion est aussi un appel au salut et ce, à l'encontre de toute autre tradition religieuse. Il ne s'agit pas d'un choix personnel relatif, mais de la reconnaissance d'une vérité absolue. Il y a, si l'on veut éviter la damnation, une "nécessité » de la conversion: on le voit dans les Stromates («epistrophē", 6,3 [448,10]) de Clément d'Alexandrie, où il est dit que Dieu force parfois la main de l'indécis en lui envoyant des signes prodigieux afin que celui-ci accueille sans plus tarder le commandement. L'idée d'une conversion suscitée par l'intervention divine aura, on le sait, une longue histoire dans la théologie chrétienne de la grâce (pensons à l'augustinienne "gratia gratum faciens", mentionnée en ouverture). Il est vrai que la conversion n'est pas toujours sereine: elle peut s'imposer de façon tourmentée, comme l'admet Origène, dans son œuvre Sur les principes $(3,1,17)$, mais ce n'est que pour en renforcer la stabilité, nous dit-il. Et c'est encore Origène qui réfléchit sur la conscience du converti dans son cheminement: il vaudrait mieux être instruit et choisir en toute connaissance de cause, mais une conversion dictée par la peur des sanctions infernales vaut toujours mieux que la damnation (Contre Celse 1,9). En ce sens, il n'est donc pas étonnant que le théologien d'Alexandrie associe epistrophē et metanoia dans un même passage de ses Homélie sur Jérémie $(1,1)$, où il réaffirme, si besoin était, que, dans ce domaine, une acceptation de la vraie foi implique en même temps un reniement de la vie précédente. Un revirement qui a des conséquences très concrètes, car le salut n'est pas exclusivement une affaire de conscience mais suppose l'entrée dans une communauté d'élus; ce qui revient à dire qu'il s'agit d'un fait historique concret, un changement de vie et de contexte. La question ne concerne pas tant la bonne ou mauvaise foi du juif et du païen dans leur affiliation religieuse, qui est parfois reconnue - et même appréciée - par les auteurs chrétiens, par exemple chez les moines du désert (Apophthegmes Série Alphabétique Olympios 1, Macaire l'Égyptien 39). Avant tout, le problème demeure l'unicité exclusive de la voie chrétienne menant au salut. Justifier cette exclusivité sur le plan uniquement moral est ardu; c'est à cette fin qu'on a souvent (plus souvent qu'on ne le pense) recours aux notions de pur et impur, notions qui encore dans le christianisme (et malgré sa tension allégorisante) - sont physiques avant même qu'éthiques (Vecoli 2007). En effet, dans ses Stromates (4,22 [311.13]), Clément affirme que la repentance est la purification parfaite de l'homme, qu'elle requiert donc le reniement complet de 
son propre passé et une condamnation de soi-même. Cette transformation, nous dit-il, est le seul moyen d'acquérir la compréhension de la vérité, d'émerger avec l'esprit «des plaisirs sensibles et des erreurs passées». En ce sens, on comprend que Clément ait pu — un peu plus tôt $(2,6[127,14])$ - définir la repentance comme une «gnose». Se convertir, se repentir, se purifier et connaître la vérité ne font qu'un. D'ailleurs, la conversion n'est complète qu'avec l'entrée effective dans la communauté chrétienne par le biais du baptême, rite - justement - de purification ${ }^{7}$. Un passage de l'Histoire des moines de la Haute Égypte (Syène) de Paphnutius (17r-17v) le montre bien: on y affirme que le chrétien est souillé par le simple contact physique d'un païen, même si celui-ci s'est déjà converti en son cœur; seul le baptême finalisera la conversion et éliminera l'impureté liée à l'adoration des idoles.

\section{Christianisme et conversion}

Il paraît donc évident que la conversion est liée à la réalité du salut ${ }^{8}$ et se situe dans un contexte religieux où celui-ci est essentiel. Sans vouloir pour autant récupérer la catégorie de «religion de salut» (qui remonte, entre autres, à E. Troeltsch et à W. Bousset), il n'est pas inutile de rappeler que certains ont cru voir dans la doctrine du salut un élément distinctif du christianisme (Kippenberg 1999, 231): eschatologie, apocalyptique et ascèse seraient les marques d'une foi qui n'a rien de naturel ou de spontané (d'où la catégorie opposée de «religion naturelle»), mais au contraire se distancie significativement du monde. Une religion de salut requiert dès lors un retournement radical du croyant par rapport à sa situation de départ (monde, société, culture ambiante...): elle exige une rupture. Les historiens considèrent les transformations religieuses de l'Antiquité tardive, avec la montée du christianisme, impossibles à appréhender sans tenir compte du phénomène de la conversion ${ }^{9}$. Celle-ci était certes connue du

7. C'est ce qu'implique l'emploi du verbe apolouō (laver), dans 1Co 6,11, en référence à ce qui a été compris comme une attestation du rite baptismal (Saxer 2000, 452). Pour une mise à jour plus récente sur l'initiation chrétienne, voir Maxwell (2008), malgré la regrettable absence de toute littérature non anglophone dans ses références.

8. Dont elle est, du moins à l'époque ancienne, le seul moyen d'accès pour un non chrétien.

9. Au-delà des pratiques quotidiennes des chrétiens, pensons seulement aux deux conversions majeures du IV ${ }^{\mathrm{e}}$ siècle: celle de Constantin, qui ouvre le siècle (312), et celle d'Augustin, qui le clôt (386). Indépendamment des débats sur l'essence et les modalités de ces conversions, elles ont toutes les deux eu un impact énorme sur 
judaïsme, mais force est de constater qu'elle prend tout son essor avec la diffusion du christianisme, soit d'un culte où l'insistance sur l'amour du prochain suscite un zèle puissant quant à son destin métaphysique, et provoque un élan missionnaire sans précédent, destiné - toujours par amour, semble-t-il (Stroumsa 2005) - à engendrer diverses formes de violence. En effet, la doctrine chrétienne insiste particulièrement sur deux aspects : d'une part, l'universalité de la foi annoncée (celle-ci n'est pas - en un premier temps du moins - un trait identitaire susceptible d'identifier une nation par rapport à une autre); d'autre part, la radicalité du changement de vie, qui exclut idéalement toute forme de compromis avec le passé culturel et religieux de provenance, et qui ne prévoit aucunement des niveaux progressifs et diversifiés de rapprochement à la nouvelle religion (comme dans le judaïsme, si l'on pense à l'idée des lois noahides — ou noachiques [Novak 1983] —, c'est-à-dire un minimum de préceptes que tout individu non juif doit observer s'il veut sa part dans le monde à venir). Dès lors s'ouvre une fracture dans le continuum de l'existence du croyant. On rétorquera (Lane Fox 1997, 328-329) que dans les anciennes communautés chrétiennes (autour du III $^{\mathrm{e}}$ siècle, par exemple) le catéchuménat durait trois ans et que la conversion était donc progressive: soit, mais le salut n'était pas accessible avant le baptême, un événement ponctuel. À cet égard, la ligne de démarcation était en effet très nette, et elle se manifestait clairement dans le rituel de l'eucharistie (véritable disciplina arcani), où les catéchumènes quittaient le rite lors de la consécration des espèces. En cela, nous adhérons à l'opinion de Arthur Darby Nock (1933), qui établissait une distinction entre "conversion» (exclusive) au christianisme et "adhésion" (non exclusive) à un autre culte. Il serait abusif de l'accuser comme il a été fait (Sanders 2002, p. 621) — de s'être conformé à une vision «idéalisée » de la conversion: de fait, Nock s'en tenait au niveau - certes abstrait, mais néanmoins significatif — de la doctrine, alors que d'autres, comme MacMullen (1984), se sont plutôt concentrés sur la pratique réelle. Or, si dans les faits la dernière ne reflète que très rarement la théorie - tout historien en est conscient - , elle n'en est pas moins parcourue d'une tension qui la motive et l'oriente, une tension dont la doctrine est en mesure de rendre compte. Nul besoin ici d'entrer dans le débat entre matérialisme et idéalisme, de choisir entre Marx et Humboldt: un peu de

l'Église, aux niveaux politique et théologique. Dans le foisonnement de la littérature sur ces questions, je renvoie encore aux classiques: Alföldi (1948), pour Constantin, et Brown (1967), pour Augustin. 
bon sens suffira. Et bien, justement, l'exclusivité - malgré tous les compromis et toutes les défaillances possibles - est un trait du christianisme qui n'appartient pas aux cultes orientaux de l'empire (souvent pris comme terme de comparaison $)^{10}$. En effet, si les chrétiens avaient été si disponibles à se glisser dans le moule de la société impériale, avec son foisonnement de traditions religieuses exotiques plus ou moins tolérées, il aurait été particulièrement malaisé d'expliquer l'histoire de leurs persécutions.

En ce qui concerne la radicalité de la conversion au christianisme, il faut aussi rappeler la relation très particulière qu'entretient le monothéisme avec la vérité religieuse. Il revient à l'égyptologue Jan Assmann (1998) d'avoir nommé le changement dans la conception de l'affiliation religieuse survenu avec l'apparition du monothéisme (dont la première manifestation remonte à la réforme religieuse d'Akhenaton, vers la moitié

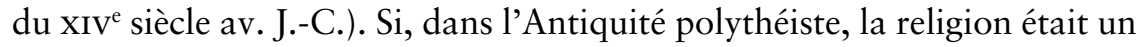
langage culturel susceptible d'être «traduit» lors de contacts avec d'autres sociétés (la religion était un véhicule de traduction des concepts fondamentaux des différentes cultures), le monothéisme introduit pour la première fois ce qu'Assmann appelle la «distinction mosaïque": d'instrument de traduction la religion devient élément de distinction intraduisible du fait de l'exclusive et universelle vérité qui y est proclamée. C'est donc avec l'introduction du concept de vérité exclusive, dans la sphère du religieux, doublé — dans le christianisme — d'un élan missionnaire sans précédent, que prend forme la réalité de la conversion: soit un passage à une nouvelle allégeance qui implique le reniement des pratiques du passé au profit de la nouvelle croyance, qui apparaît comme l'unique vraie.

En ce qui concerne l'universalisme de la foi chrétienne, il s'agit là d'un trait qui remonte à ses origines: je me contenterai de mentionner la célèbre Épître à Diognète (5-6). Les premiers chrétiens, selon ce texte, constituent l'âme du monde et ne s'identifient à aucune nation; ce qui exclut d'emblée toute référence à une ethnicité quelconque dans l'idée de conversion au christianisme. Et pourtant, quand l'Empire romain devient chrétien après la conversion de l'empereur Constantin (272-337) au début du IV siècle, une équivalence entre chrétien et romain (c'est-à-dire impérial) s'impose progressivement. On le voit dans le destin de l'Église nestorienne,

10. Voir, par exemple, l'invocation d'Isis dans Apulée (Métamorphoses XI,1,1-XI,5,5): Lucius s'adresse à la divinité en l'appelant avec les noms de tous ses équivalents dans la Méditerranée ancienne: la même chose serait tout simplement inconcevable pour un chrétien. 
contrainte de se distancier de l'Église impériale pour éviter que les autorités perses ne l'identifient avec une religion étrangère, associée à un empire ennemi (Leroy 1972, Hage 1997, Brock 1982).

\section{Secte et Église: quelle conversion?}

Malgré l'indéniable universalisme de l'Épître à Diognète, la conviction des autorités perses en ce qui concerne l'existence d'un lien entre christianisme et nation (lien qui aura une longue histoire dans l'Orient orthodoxe) n'est pas injustifiée. En effet, après la faveur accordée par Constantin aux chrétiens, l'Église évolue en institution impériale et s'élève par la suite au rang de religion d'État. Par conséquent, l'appartenance à la religion chrétienne devient une réalité sociale de plus en plus donnée pour acquise: en témoigne l'apparition, à cette époque, du baptême des enfants (pédobaptisme) ${ }^{11}$. Or, c'est justement sur la base des transformations qui ont eu lieu au IV $^{\mathrm{e}}$ siècle que le sociologue Ernst Troeltsch (1919) a pu, au début du $\mathrm{xx}^{\mathrm{e}}$ siècle, appliquer à l'histoire du christianisme la célèbre distinction entre "secte " (communauté d'élection, en rupture avec le monde ambiant, à laquelle on adhère en vertu d'un choix) et «Église » (institution entretenant un rapport de symbiose avec la société et religion dans laquelle le croyant naît $)^{12}$. En simplifiant, le christianisme serait né comme «secte» et serait peu à peu devenu une "Église ", particulièrement à la suite du virage constantinien. Ce qui nous intéresse ici, de la distinction de Troeltsch, c'est que la conversion, dans une religion de type sectaire, n'entretient aucun rapport avec l'ethnicité, car il ne s'agit pas de faire partie d'un nouveau peuple au sens ethnique du terme, mais d'adhérer à un credo à la fois élitiste et universel. Cela changera, justement, à mesure qu'avanceront les processus d'institutionnalisation et d'impérialisation de l'Église. On peut d'ailleurs se demander si, dans le nouveau contexte culturel et social post-constantinien, il est encore légitime de parler de conversion, car l'accès à la foi chrétienne se

11. Il faut signaler qu'il y a débat sur l'époque de diffusion de cette pratique (et ce, aussi pour des raisons de polémique interconfessionnelle). Les historiens, toutefois, semblent considérer qu'il est difficile de prouver la diffusion du pédobaptisme avant le $\mathrm{IV}^{\mathrm{e}}$ siècle, en tout cas pas pour des cas autres que de danger de mort de l'enfant.

12. Comme il a été remarqué (Steeman 1975), Troeltsch utilisait ces notions en référence à des moments historiques bien précis. Le débat sur la distinction Église-secte a été influencé par l'emploi de ces notions comme catégories abstraites intemporelles, une démarche qui a commencé avec leur transposition (Niebuhr 1929) dans le monde religieux contemporain (notamment américain) avec - de plus - l'ajout de nouvelles considérations d'ordre axiologique. 
fait de plus en plus par le processus normal de socialisation. En effet, certains déploreront de voir entrer dans l'Église des fidèles d'une foi douteuse (Salamito 1995, 683): Eusèbe mentionnera des hypocrites qui agissent par intérêt (Eusèbe de Césarée, Vita Constantini 4,54) et Augustin, des convertis par nécessité (Augustin d'Hippone, Sermones Morin 1,1). La conversion, plus qu'un choix individuel spontané, commence à désigner l'inclusion de populations entières — d'abord rurales, mais par la suite étrangères - à l'intérieur d'un écoumène méditerranéen de plus en plus chrétien. C'est à cette période que théologie missionnaire et politique impériale se rapprochent et finissent par se rejoindre.

\section{La conversion dans le monachisme primitif}

En ce milieu chrétien en voie d'institutionnalisation, le phénomène du monachisme connaît son essor (Desprez 1998; Filoramo 2010). Ce nouveau genre de vie religieuse réaffirme la nécessité d'une conversion réelle et individuelle à l'intérieur d'une société de plus en plus christianisée, donc perçue comme étant trop compromise avec «le monde». Il a été dit que nous assistons, à partir du IV siècle, à une véritable «crise d'identité » des chrétiens (Markus 1990, 19-43), ceux-ci éprouvant de croissantes difficultés à se distinguer du reste de la société (à laquelle, de fait, ils correspondent de plus en plus): en cet état de tiédeur du corps des croyants, l'ascétisme des plus fervents finit par s'imposer comme la caractéristique distinctive du christianisme. Or, le monachisme est justement constitué de virtuoses de l'ascétisme, ce qui lui permet de transformer le sens de la conversion en lui rendant sa pertinence sur le plan individuel: là où s'estompe le choix individuel d'entrée dans le christianisme s'affirme le choix de suivre un parcours d'excellence spirituelle ${ }^{13}$. La conversion étant l'entrée de l' "autre » dans une communauté de foi - ce qui pose problème dans une société de plus en plus christianisée où l' " autre » religieux est en voie de disparition -, la conversion monastique situe l'altérité à l'intérieur de chaque homme, rétablissant ainsi la dimension à la fois universaliste et personnelle de l'évènement. Car, dans une perspective ascétique, l'altérité qui doit être ramenée à l'uniformité monothéiste n'est plus seulement celle de l'appartenance à une religion fausse et mensongère, mais plutôt celle d'une intériorité agitée par de multiples tendances centrifuges, où les intentions les plus

13. Il s'agit là d'un processus graduel qui peut donner lieu à des épisodes parfois contradictoires. 
disparates et indisciplinées sont à réorienter vers l'unité et l'unicité d'une parfaite superposition entre volonté humaine et volonté de Dieu. En d'autres termes, le complexe - perçu comme équivalent de la duplicité (dipsychia) et donc relevant de la dispersion et du faux — doit revenir à la simplicité (aplotēs) (Lane Fox 1997, 379), selon un processus de concentration sur ce qui seul est vrai et solide. C'est ce que les moines nomment «monotropia» (Guillaumont 1979, 51-54), soit l'être un et unifié avec Dieu.

Si on revient aux catégories de Troeltsch, le monachisme primitif semblerait récupérer la dimension sectaire, pour ainsi dire, du christianisme, rétablissant l'élément du "choix » nécessaire à toute adhésion sincère, ainsi que la tension entretenue avec un monde jugé de façon généralement négative, et avec l'Église qui s'y installe trop confortablement. Dans ce contexte, il n'est pas étonnant que certains auteurs évoquent une éventuelle tendance du monachisme des origines (dans sa déclinaison la plus institutionnalisée, c'est-à-dire le cénobitisme pachômien) à se constituer comme ecclesiola in ecclesia, soit une "petite Église" à part, à l'intérieur de la grande Église (Baumeister 1977, Gould 1986). Bref, le monachisme en un premier temps redélocalise le christianisme en dehors de l'institution ecclésiastique (Goehring 1999, Caner 2002), et ce, dans le but de cesser toute compromission avec la société profane (donc avec l'institution impériale et, s'il en fut, avec toute considération liée à la nationalité).

Ce changement dans la conception de la conversion chez les moines en produit un autre, tout aussi significatif: dans la doctrine ascétique des anachorètes, la conversion cesse d'être un évènement ponctuel qui, indiquant le moment d'entrée dans une nouvelle communauté de foi, établit de façon nette un avant et un après dans la biographie du fidèle. Au contraire, la conversion - pour le moins ad intra - est de plus en plus conçue comme un effort quotidien de détachement qui ne peut être définitif en raison des résistances de l'esprit humain, toujours leurré par les tentations pécheresses. Il s'agit donc d'un choix — ici aussi parfois exprimé avec l'image des deux voies ${ }^{14}$ - qui se répète à chaque moment de la vie spirituelle, un choix qui n'est confirmé de façon définitive que par la mort. C'est là que se situe l'élément distinctif de ce qu'on pourrait appeler le tournant monastique.

14. «Il y a donc deux voies, l'une de la vie, et l'autre de la mort [...] Que celui qui veut entrer dans le Royaume en garde les œuvres» dans Isaïe de Scété, Recueil ascétique 21, 1.3. 
Pour expliquer comment cela a pu se produire, il faut commencer par souligner au moins deux points concernant l'émergence du monachisme chrétien au $\mathrm{IV}^{\mathrm{e}}$ siècle.

\subsection{Excellence du monachisme chrétien}

Tout d'abord, il est à noter que le monachisme s'impose très tôt comme une élite religieuse. Se convertir «sérieusement » au christianisme, à partir de cette époque, c'est se convertir au monachisme: l'assouplissement de la discipline chrétienne - du fait de sa diffusion à l'intérieur de l'Empire et de son caractère de plus en plus obligatoire pour l'ensemble de la citoyenneté - crée le besoin d'une réaffirmation de ses exigences profondes.

Il convient de citer à ce propos le récit de la conversion du premier moine chrétien selon la tradition, Antoine (251-357). Sa biographie, très probablement rédigée par l'évêque d'Alexandrie, Athanase, en 356 apr. J.-C., décrit sa conversion comme étant provoquée par la lecture à l'église d'une péricope évangélique qui insiste justement sur les renonciations requises pour se mettre à la suite de Jésus. Voici donc ce que raconte la Vie d'Antoine (2, 3-4):

Le cœur occupé de ces pensées, il entra dans l'église, et il se trouva qu'on lisait justement l'Évangile; il entendit le Seigneur dire au riche « Si tu veux être parfait, va, vends tout ce que tu possèdes et donne-le aux pauvres, et viens, suis-moi, et tu auras un trésor dans les cieux (Mt 19,21)». Antoine, comme si le souvenir des saints, qu'il venait d'avoir, lui était venu de Dieu et comme si la lecture avait été faite pour lui, il sortit aussitôt de la maison du Seigneur.

... et Antoine fit ce que lui ordonnait l'Évangile.

Le bouleversement présenté dans ce récit nous intéresse particulièrement parce que son acteur est issu d'une famille chrétienne. Donc, chrétien, il l'est lui aussi avant d'entendre les mots qui lui inspireront le choix d'une vie consacrée à l'ascétisme. On pourrait parler, dans le cas d'Antoine, d'une «seconde conversion» (Pinard de la Boullaye 1953, 2259-2265). L'expression vient de Clément d'Alexandrie (Stromates 7,10), qui distinguait entre une première conversion, du paganisme à la foi, et une deuxième, de la foi à la gnose (terme avec lequel il indiquait une connaissance plus profonde des mystères chrétiens). L'articulation du processus de conversion répond sans doute au besoin de conserver à celle-ci un sens et une place dans la spiritualité de tous ceux pour qui l'adhésion au christianisme est 
désormais considérée comme acquise. Cette conception est destinée à un succès durable: une réflexion sur la "seconde conversion" se retrouvera - et ce n'est pas un hasard - dans le catholicisme post-tridentin, notamment chez les jésuites Bernardino Rosignoli (1547-1613) ${ }^{15}$ et Antoine Le Gaudier (1572-1622 $)^{16}$. Mais le texte le plus frappant, quand on le compare à ceux des premiers moines, est issu de la grande synthèse de spiritualité ignatienne produite par Louis Lallemant (1587-1635), un autre jésuite: "Il arrive d'ordinaire deux conversions à la plupart des saints et aux religieux qui se rendent parfaits; l'une par laquelle ils se dévouent au service de Dieu, l'autre par laquelle ils se donnent entièrement à la perfection » (Pottier 1936, 67).

Tout cela nous montre quel a été l'impact sur le long terme de l'avènement du monachisme dans le cadre du sujet qui nous intéresse. L'entrée dans ce qui deviendra plus tard un véritable tagma (ordre: De Vogüé 2003) - donc une institution au même titre que tout autre ordre régulier - était en effet perçue comme une conversion réelle, encore qu'une simple étape, la première, vers la perfection spirituelle. En ce sens, le sérieux de l'engagement ascétique n'attire pas que ceux qui adhèrent depuis leur naissance à la foi en Jésus, mais aussi bien les païens encore présents dans la société impériale: une vocation forte ne pouvait désormais plus se satisfaire d'une simple entrée dans le christianisme des masses. On se rappellera qu'à l'origine l'acceptation dans un monastère correspondait souvent à l'adhésion au christianisme: en témoigne, dans les couvents de la congrégation cénobitique des Tabennésiotes, l'usage de former dans le catéchuménat et de baptiser les novices (Veilleux 1968, 198-225). Le fondateur, Pachôme (292-346), était lui-même issu du paganisme et s'était converti presque directement à la vie monastique: rien d'étonnant donc qu'il fut prêt à recevoir des païens dans sa communauté. Chez lui, la formation chrétienne était une formation monastique. D'autre part, au fil des décennies, les recrues chrétiennes augmentent au point où l'on en arrive à considérer la vie monastique comme un «second baptême » ou, du moins, est-ce la formule qu'utilisera Jérôme de Stridon pour la désigner (Dekkers 1958). Dans tout cela, le chrétien ordinaire est de moins en moins considéré: nous sommes en effet au début d'un processus qui mènera à une distinction nette entre laïcs et religieux (Faivre 1984), distinction qui présente des analogies

15. De disciplina christiance perfectionis, Ingolstadt 1600 , lib.1, c.3, p. 16 ; c.4, p. 24

16. De perfectione vitce spiritualis, $2^{\mathrm{e}}$ éd., Paris $1856-1858, \mathrm{t} .1$, p. 216-222, trad. Bizeul, Bruxelles 1908, t.1, p. 298-306. 
avec celle qui avait cours dans un autre milieu religieux particulièrement important pour l'époque, le manichéisme, lui-même à son tour influencé par l'organisation des communautés bouddhistes (Tardieu 1997, p. 76-77).

L'évolution de la vie religieuse à la suite de la diffusion du monachisme semble avoir introduit une nouvelle perception de la conversion, comprise non plus seulement comme adhésion à la foi chrétienne, mais comme recherche de perfection spirituelle. Quoi qu'il en soit de la locution "second baptême ", de sa diffusion et de son utilisation réelle, il est certain que la profession monastique devait désormais apparaître comme le «retournement» le plus significatif dans la vie d'un fidèle.

\subsection{Le nouveau rapport avec le passé}

Cela dit, du point de vue de ce virtuose de la religion qu'est le moine, n'est pas sauvé qui est chrétien ni même qui pratique l'ascèse. Par conséquent, l'événement de la conversion n'établit pas un avant et un après aussi net qu'auparavant. Certes, la torsion spirituelle ouvre une dimension nouvelle, mais elle ne fournit aucune garantie quant au salut du concerné. La spiritualité monastique radicalise, si possible, les enjeux: le passé par rapport auquel on mesure l'exigence de la conversion (et donc la tension vers le salut) n'est plus simplement celui d'avant l'entrée dans le christianisme. La dichotomie simplifiée paganisme-christianisme est, sur ce plan, en perte de pertinence. Le passé qui obsède le solitaire, c'est le hier de chaque jour, vécu par l'irrémédiable pécheur qu'est l'être humain. Un passage des Apophtegmes, synthèse particulièrement efficace de la doctrine monastique orientale ancienne, nous l'explique: "Ainsi en va-t-il pour qui a pratiqué la vie monastique. S'il succombe à la tentation, et se convertit ${ }^{17}$, il dispose de nombreux moyens: la méditation, la psalmodie et le travail manuel...» (Apophtegmes Série Systématique V,22,13) ${ }^{18}$. Chaque tentation, si l'on y cède, requiert subséquemment une conversion. Le verbe epistrephō indique maintenant un retournement du péché à la vertu. L'enjeu reste toujours le salut: "Car l'enjeu en est pour eux [les disciples], s'ils se convertissent [epistrephō] et se tournent vers Dieu, le salut de leur âme" (Apopthegmes Série Systématique XVIII,46,99). En quête d'un précédent biblique prestigieux pour une telle opération de transformation sémantique du concept

17. Le verbe utilisé pour indiquer la conversion est précisément epistrefō.

18. Voir aussi X,62,2; X,100,19; XVII,16,3; XVIII,26,93; XVIII,46,99.103 : chaque fois, on se "convertit (epistrefō)» du péché. 
de conversion, l'auteur récupère le célèbre passage d'Ézéchiel $(18,23)$ : «Je ne veux pas la mort du pécheur, mais qu'il se convertisse et vive» (cité dans Apophtegmes Série Systématique XVIII,26,93). Toutefois, il est à noter que, dans la Septante, le verbe utilisé est apostrephō (qui insiste plutôt sur le mouvement d'abandon du mal que l'on commettait avant), alors que, dans la citation proposée dans les Apophtegmes, on le remplace par epistrephō, ce qui montre bien la volonté de redéfinir la signification de "conversion". Quant aux péchés auxquels on renonce, il ne s'agit pas nécessairement de ceux qui sont encadrés par l'Église institutionnelle, pour lesquels sont établies une confession et une pénitence. La tentation et la chute, dans le monachisme ancien, peuvent se manifester par une simple pensée (les fameux logismoi, le pire ennemi des Pères du désert). À un moine qui décide de quitter la vie monastique, découragé à cause des tentations persistantes qui l'affligent, Dieu promet: «Les neuf années pendant lesquelles tu as été tenté ont été pour toi des couronnes; retourne (epistrepson) donc où tu étais et je vais te décharger de ces pensées (logismōn)» (Apopthegmes Série Systématique VII,51,7). Retourner (epistrephō) au lieu de l'ascèse, c'est revenir du péché à la vertu, se libérer des pensées mauvaises, se convertir (epistrephō). Conversion et repentance ont tendance à se superposer, mais il faut néanmoins souligner - question de détail non négligeable - que c'est la première (epistrophēe), comprise comme le retour sur la bonne voie après l'égarement, qui ouvre la porte à la deuxième

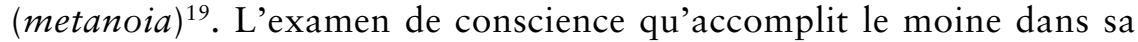
cellule est infiniment plus exigeant par rapport à ce qui est demandé au chrétien ordinaire: il demande une "attention à soi " (prosochē) tout à fait spéciale (Miquel 1986, 249-258). À ce propos, on a à juste titre fait le rapprochement avec l'examen de conscience, dans la pratique philosophique ancienne (Hadot 2002).

Si chaque tentation est susceptible de devenir pierre d'achoppement, il est aisé de comprendre que la conversion, dans la doctrine ascétique des premiers moines, n'a plus rien d'un évènement ponctuel et unique. Il s'agit, comme nous l'avons dit, d'un effort continu, expression d'une inébranlable volonté de se relever après chaque chute. De sorte que la conversion se manifeste dans une tension perpétuelle, finalisée à une croissance spirituelle qui n'aboutit jamais et qu'on ne mesure donc pas sur les étapes

19. Sur la repentance dans l'Antiquité tardive, avec une attention particulière aux textes monastiques orientaux, voir Torrance (2013). 
atteintes - car la perfection n'appartient pas, de toute façon, à l'humanité post-lapsaire -, mais sur le fait d'être en mouvement.

Un extrait de la Vie d'Antoine explique de façon très claire ce nouveau rapport au passé du pécheur:

À cause de cela aussi que chacun se persuade de ne pas se laisser aller à la négligence, surtout s'il considère qu'il est serviteur du Seigneur et obligé de servir son maître. De même en effet qu'un serviteur n'osera pas dire: «Puisque j'ai travaillé hier, je ne travaille pas aujourd'hui », et qu'il ne mesure pas le temps passé pour cesser de travailler les jours suivants, mais que chaque jour, comme il est écrit dans l'Évangile, il montre la même ardeur afin de plaire à son maître et ne pas courir de risque; de même, nous devons nous aussi persévérer chaque jour dans l'ascèse, sachant que, si nous sommes négligents un jour, le Seigneur ne nous pardonnera pas en raison du temps passé, mais s'irritera contre nous en raison de notre négligence. Ainsi l'avons-nous appris chez Ézechiel ${ }^{20}$; ainsi Judas, pour une seule nuit, perditil le labeur du temps passé.» (Vie d'Antoine 18,1-3)

Pachôme s'exprime lui aussi de façon très efficace dans sa Catéchèse à propos d'un moine rancunier (26): "Cette année est pareille à l'année passée et aujourd'hui est comme hier. Tant que tu restes négligent, il n’y a pas de progrès pour toi.»

\section{L'éternel présent: la conversion comme une progression sans fin}

La conversion devient une progression dépourvue d'un terme en cette vie, une ascension incessante. L'adhésion à l'exemple du Christ - l'essence d'une véritable conversion - est l'adhésion au parcours d'un Crucifié, dont le terme en ce monde ne peut être que la mort. Isaïe de Scété ( $v^{\mathrm{e}} \mathrm{s}$.), grand théoricien du monachisme ancien, interprétera d'ailleurs l'itinéraire ascétique du moine comme une «montée sur la croix». La mortification et la retraite, devises du moine, sont considérées à l'instar d'un baptême et tracent un parcours sur lequel s'engage seul celui qui a fermé la bouche des "Sadducéens incroyants» (ce qui signifie qu'il faut posséder la foi, soit se convertir). Cela équivaut à dire, encore une fois, que la discipline monastique ne peut exister sans la conversion (Isaie de Scété, Recueil ascétique 13,2):

Voici ce qu'est le baptême: la mortification dans l'humilité et la retraite. Il est écrit en effet de Jean que son manteau était en poils de chameau, qu'une

20. Ez 3,20: «Lorsque le juste se détournera de sa justice pour commettre le mal [...], il mourra de son péché et on ne se souviendra plus de la justice qu'il a pratiquée. » 
ceinture de peau ceignait ses reins (Mt 3,4), et qu'il était dans le désert; c'est le signe de la mortification. Celle-ci purifie d'abord l'homme qui, s'il travaille, l'acquiert; et, quand il l'a acquise, il est libre de monter sur la croix. La croix est le signe de l'immortalité qui doit arriver lorsqu'a été d'abord fermée la bouche des Pharisiens et des Sadducéens, - les Sadducéens sont l'image de l'incrédulité.

Nous l'avons dit, ce parcours qu'est la montée sur la croix (pour Isaïe de Scété) ne peut être achevé avant la mort, car l'imitation du Christ est présentée comme l'adhésion à un modèle de perfection absolue auquel il est impossible de se conformer en tout et pour tout. On ne peut que s'en rapprocher un peu plus chaque jour. Paul de Tamma ( $\mathrm{V}^{\mathrm{e}}$ siècle), un moine copte de cette époque, l'exprime explicitement dans son De Cella (44-47): «Dieu est infini. Il n'y a pas de mesure pour le sage qui sied dans sa cellule. La mesure du sage est le Seigneur ", qui est lui-même infini... C'est donc le mouvement qui compte, non pas les étapes franchies (bien que celles-ci existent): la progression est instamment recalibrée sur l'infini du but à atteindre. Seule l'entrée dans l'infini, soit la mort, peut donc fixer un terme à cette tension.

La conversion du moine place celui-ci en présence de Dieu, dans une dimension temporelle nouvelle où seul le présent, un présent liturgique, compte ( «afin que tu sois ainsi toujours comme faisant la synaxe ", dit Isaïe de Scété, Recueil ascétique 27,7). L'alternance sacré-profane ${ }^{21}$, situation normale de toute forme de vie religieuse ${ }^{22}$, est ici brisée en faveur d'une entrée définitive dans le temps de Dieu ou, du moins, c'est le but que les solitaires visent à atteindre par leurs pratiques ascétiques. La conversion, en tant que mouvement de celui qui se tourne vers Dieu, n'est jamais complète: il faudrait pour cela sortir définitivement du temps profane, une action que le moine entreprend, mais qui ne peut être achevée en ce bas monde. L'epistrophē du moine est un retournement opéré pour se mettre en présence de Dieu et s'en rapprocher le plus possible. Par un travail sur le corps (l'ascèse) s'opère un mouvement de l'esprit qui ouvre une dimension mystique nouvelle. Le biographe de Pachôme insiste particulièrement

21. Alternance analysée, en rapport avec la catégorie de l'espace dans le monachisme ancien, par Vecoli (2010).

22. Les catégories de sacré et de profane sont certes souvent contestées, et la bibliographie sur la question est très vaste. Sans vouloir entrer dans le débat, il suffira de préciser que, dans ce cas, qui est celui d'une utilisation heuristique, elles nous apparaissent tout à fait pertinentes. 
sur cet aspect quand il explique ce que signifie avoir le don de la vision divine (Vie grecque de Pachôme 48):

Du moins ont-ils [les saints, donc les moines] sans cesse un autre pouvoir de vision, celui de voir le Seigneur: ce que l'un d'eux nous a signifié quand il dit (Ps 15,8): «En avant de moi j'ai vu le Seigneur devant ma face tout le temps ». Un individu n'est pas jugé en ce qu'il ne voit pas les choses cachées: mais il est jugé s'il porte la ressemblance de ceux qui dans le psaume $(53,5)$ sont condamnés par l'Esprit, lorsqu'il dit: "Ils n'ont pas mis Dieu en avant d'eux devant leur face».

Dans la doctrine monastique, la conversion perd son intérêt d'événement social et finit par traduire une tension intime qui aboutit à une théorie mystique inédite dans la théologie chrétienne. "Conversion» signifie maintenant un parcours d'ascension spirituelle qui place le croyant en présence de Dieu, une présence qu'il doit s'efforcer de maintenir en tout temps. Pour ce faire, il lui faut assumer le contrôle de la dimension temporelle: l'oraison et la mortification deviennent les armes pour la conquête du présent, un éternel présent qui est celui de la vie en Dieu (Vecoli 2006, 85-88). L'échec, dans une conversion ainsi conçue, est donc compris comme l'incapacité à pénétrer dans cette nouvelle phase temporelle que l'on pourrait qualifier de liturgique. Antoine le dit bien dans une de ces Lettres $(6,3)$, où il pleure ceux qui se sont perdus «du fait qu'ils ont trouvé trop long le temps et se sont découragés".

Le passé ne relève plus de la chronobiographie du converti, mais représente une condition de déchéance prototypique que l'on s'efforce de quitter définitivement. Et le futur fusionne en quelque sorte avec le présent: ce n'est plus l'insupportable attente de la fin du labeur, mais la fixation définitive dans l'au-delà d'un présent qui se situe dès maintenant hors de toute temporalité profane. J'en reviens ici à la Vie d'Antoine:

Lui-même ne se souvenait pas du temps passé, mais jour après jour, comme s'il débutait dans l'ascèse, il renforçait ses efforts pour progresser, en se répétant continuellement le mot de saint Paul: «Oubliant ce qui est derrière moi, et tendu de tout mon être (epekteinómenos) vers ce qui est en avant (Phil 3,13) ». Il se souvenait aussi de la parole du prophète Élie: «Le Seigneur est vivant, devant lequel je me tiens aujourd'hui ${ }^{23}$ (3Rois 17,$1 ; 18,15$ )» Il faisait remarquer qu'en disant "aujourd'hui ", Élie ne mesurait pas le temps passé, mais, comme s'il débutait constamment, s'efforçait chaque jour de se

23. Hors citation biblique: c'est un ajout de V.A. qui souligne la dimension temporelle de la présence devant Dieu. 
montrer à Dieu tel qu'il faut paraître devant Dieu: pur de cœur et prêt à obéir à sa volonté, et à nulle autre. (Vie d'Antoine 7,11-13)

Cette dimension mystique redéfinit l'événement de la conversion en modifiant la perception du temps dans la vie spirituelle du moine. Pour bien l'exprimer, Athanase reprend les mots de Paul qui constitueront le fondement de la mystique chrétienne successive, des mots qui parlent d'une course qui n'aboutit jamais, d'une tension toujours inachevée à laquelle Grégoire de Nysse (Homélies sur le Cantique des Cantiques, M.44.888a) donnera le nom d' «épectase » (epectasis) ${ }^{24}$. Le terme, en grec, signifie précisément «extension, allongement ". Avec Grégoire de Nysse et l'épectase, s'ouvre donc la théorie contemplative chrétienne (Daniélou 1944), qui va établir un lien entre conversion et mystique.

Ce que le monachisme accomplit en associant conversion, purification et dimension mystique, c'est de christianiser, en un temps devenu propice à l'Église, une notion de conversion qui - à certains égards ${ }^{25}$ - existait déjà dans la philosophie grecque. Car - et je ne fais que le mentionner en ce lieu - c'est bien chez Plotin (205-270) que la conversion se définit (de la même manière que chez nos moines) comme processus de réorientation du soi qui a lieu dans l'intériorité de l'individu, là où s'opère justement le retour à Dieu par une purification qui permet enfin de le voir («L'expérience mystique apparaît ici comme un retour de l'âme à son origine ", Hadot 1997, 95). Il est donc tout à fait opportun de conclure avec les mots du philosophe (Ennéades I,2,4):

Il faut donc, après avoir purifié l'âme, l'unir à Dieu; or, pour l'unir à Dieu, il faut la tourner vers lui. Cette conversion (epistrophēe ne commence pas à s'opérer après la purification; elle en est le résultat même. La vertu de l'âme ne consiste pas alors dans sa conversion, mais dans ce qu'elle obtient par sa conversion. Or qu'obtient-elle? L'intuition de l'objet intelligible, son image produite et réalisée en elle, image semblable à celle que l'œil a des choses qu'il voit.

24. Cette tension est d'ailleurs évoquée (tendimus) par l'extrait d'Augustin, cité plus haut (De Civitate Dei X,4).

25 «À certains égards» est ici une précision essentielle. 


\section{Références}

\section{Sources}

Antoine, Lettres:

GARITTE, G. (1955), Lettres de saint Antoine. Version géorgienne et fragments coptes, Louvain, Peeters (CSCO 148-149).

APOPHTEGMES DES PÈRES :

Série Alphabétique: Migne, J.P., Patrologia Groeca LXV.

Série Systématique: Guy, J.-Cl. (1993-2005), Les Apophtegmes des pères, Paris, Cerf (SC 387, 474, 498).

Athanase D'Alexandrie, Vie d'Antoine:

Bartelink, G.J.M. (1994), Athanase d'Alexandrie. Vie d'Antoine, Paris, Cerf (SC 400).

IsAÏE DE SCÉTÉ, Recueil ascétique:

Regnault, L., De Broc, H. (1976²), Abbé Isaïe. Recueil ascétique, Bégrolleen-Mauges, Abbaye de Bellefontaine.

PACHÔME, Écrits :

Lefort, L.Th. (1956), CEuvres de S. Pachôme et de ses disciples (CSCO 159160 : texte copte et traduction française), Louvain, Peeters.

Paphnutius, Histoires des moines de la Haute Égypte:

Wallis Budge, E.A. (1977), Coptic Texts. Volume V. Miscellaneous Texts in the Dialect of Upper Egypt, New York, AMS Press, p. 432-495.

Paul de Tamma, CEuvres:

Orlandi, T. (1988), Paolo di Tamma. Opere, Roma, C.I.M..

VIE DE PACHÔME:

Halkin, F. (1932), Sancti Pachomii Vitce Gracce, Bruxelles, Société des Bollandistes (SH 19).

Festugière, A-J. (1962-1963), Les moines d'Orient IV/2. La première vie grecque de saint Pachôme, Paris, Cerf.

\section{Littérature secondaire}

Alföldi, A. (19692) [1948], The Conversion of Constantin and Pagan Rome, Oxford, Clarendon Press. 
Assmann, J. (1998), Moses der Ägypter. Entzifferung einer Gedächtnisspur, München, Hanser.

Attridge, H. W. (2000), "Didache", dans L. H. Schiffman et J. C. VanderKam, Encyclopedia of the Dead Sea Scrolls, Oxford, Oxford University Press, p. 204-205.

Baumeister, T. (1977), «Die Mentalität des frühen ägyptischen Mönchtums. Zur Frage der Ursprünge des christlichen Mönchtums ", Zeitschrift für Kirchengeschichte, 88, p. 145-160.

Borgeaud, P. (2004), Aux origines de l'histoire des religions, Paris, Seuil.

Bradbury, S. (1996), Severus of Minorca. Letter on the Conversion of the Jews, Oxford, Clarendon Press.

Brock, S. P. (1982), "Christians in the Sasanian Empire. A Case of Divided Loyalties ", dans S. Mew, Religion and National Identity: papers read at the nineteenth Summer meeting and the twentieth Winter meeting of the Ecclesiastical History Society, Oxford, Blackwell, p. 1-19.

Brown, P. (1967), Augustine of Hippo. A Biography, London, Faber and Faber.

Caner, D. (2002), Wandering, Begging Monks. Spiritual Authority and the Promotion of Monasticism in Late Antiquity, Berkeley/Los Angeles/ London, University of California Press.

Daniélou, J. (1944), Platonisme et théologie mystique. Essai sur la doctrine spirituelle de Grégoire de Nysse, Paris, Aubier.

Dekkers, E. (1958), "Profession - Second baptême. Qu'a voulu dire S. Jérôme?", Historisches Jahrbuch des Görresgesellschaft, 77, p. 91-97.

De Vogüé, A. (2003), "La législation de Justinien au sujet des moines ", Revue Mabillon, n.s.14, p. 139-151.

Desprez, V. (1998), Le monachisme primitif. Des origines jusqu'au concile d'Éphèse, Bégrolles-en-Mauges, Abbaye de Bellefontaine.

Duhaime, J. (2000), "Dualism», dans L. H. Schiffman et J. C. VanderKam, Encyclopedia of the Dead Sea Scrolls, Oxford, Oxford University Press, p. 215-220.

Faivre, A. (1984), Les laïcs aux origines de l'Église, Paris, Centurion.

Filoramo, G. (2004), Che cos'è la religione. Temi, metodi, problemi, Torino, Einaudi (tr.fr.: 2007, Qu'est-ce que la religion? Thèmes, méthodes, problèmes, Paris, Cerf). 
Morcelliana.

(2010), Monachesimo orientale. Un'introduzione, Brescia,

Goenring, J. E. (1999), «Hieracas of Leontopolis: The Making of Desert Ascetic", Ascetics, Society, and the Desert. Studies in Early Egyptian Monasticism, Harrisburg, Trinity Press International, p. 110-133.

Gould, G. (1986), «Pachomios of Tabennesi and the Foundation of an Independent Monastic Community", dans W. J. SHeIls et D. Woop, Voluntary Religion, London, Blackwell, p. 15-24.

Goffi, T. (2001), "Conversion", dans S. De Fiores et T. Goffi, Dictionnaire de la vie spirituelle, Paris, Cerf, p. 197-201.

Guillaumont, A. (1979), "Monachisme et éthique judéo-chrétienne », Aux origines du monachisme chrétien. Pour une phénoménologie du monachisme, Bégrolles-en-Mauges, Abbaye de Bellefontaine, p. 47-66.

Надот, P. (1997), Plotin ou la simplicité du regard, Paris, Gallimard. Michel.

Hage, W. (1997), Syriac Christianity in the East, Kottayam, St. Ephrem Ecumenical Research Institute.

Kippenberg, H. G. (1999) [allemand 1997], À la découverte de l'Histoire des Religions. Les sciences religieuses et la modernité, Paris, Salvator.

Lampe, G. W. H. (1961), A Greek Patristic Lexicon, Oxford, Clarendon Press.

Lane Fox, R. J. (1997) [anglais 1986], Païens et chrétiens. La religion et la vie religieuse dans l'empire romain de la mort de Commode au concile de Nicée, Toulouse, Presse Universitaires du Mirail.

Leroy, J. (1972), «Les Églises orientales non orthodoxes ", dans H. C. Puech, Histoire des religions. Vol. 2, Religions de salut (monde méditerranéen et Proche-Orient). Religions constituées (Occident), Paris, N.R.F. (Encyclopédie de la Pléiade), p. 869-910.

Lonergan, B. J. F. (1971), Doctrinal Pluralism, Marquette University Press, Milwaukee. (1972), Method in Theology, Herder and Herder, New York.

MacMullen, R. (1984), Christianizing the Roman Empire (A.D. 100-400), New Haven, Yale University Press.

Markus, R. A. (1990), The End of Christianity, Cambridge, Cambridge University Press. 
MaXwell, E. J. (2008), "Christian Initiation", dans S. A. Harvey et D. G. Hunter, The Oxford Handbook of Early Christian Studies, Oxford, Oxford University Press, p. 693-710.

Miquel, P. (1986), Lexique du désert. Étude de quelques mots-clés du vocabulaire monastique grec ancien, Bégrolles-en-Mauges, Abbaye de Bellefontaine.

Moloney, F. J., Lawrence, R. T. et Kirwin, G. F. (2003), "Conversion», dans T. Carson et J. Cerrito, New Catholic Encyclopedia. Second Edition. 4, Com-Dyn, Detroit, Thomson Gale, p. 231-241.

Moreschini, C. et Norelli, E. (2000) [italien 1995], Histoire de la littérature chrétienne ancienne grecque et latine. 1. De Paul à l'ère de Constantin, Genève, Labor et Fides.

Niebuhr, H. R. (1929), The Social Sources of Denominationalism, New York, Henry Holt and Company.

Nock, A. D. (1933), Conversion. The Old and the New in Religion from Alexander the Great to Augustine of Hippo, Oxford, Oxford University Press.

Novak, D. (1983), The image of the non-Jew in Judaism: an historical and constructive study of the Noahide Laws, New York, E. Mellen Press.

Pinard de la Boullaye, H. (1953), "Conversion", dans Dictionnaire de Spiritualité Mystique et Ascétique. T. II (Cabasilas-Cyrille de Scythopolis), Paris, Beauchesne, p. 2224-2265.

Pottier, A. (1936), Louis Lallemant. Doctrine spirituelle, Paris, Desclée de Brouwer.

Rambo, L. R. et Farhadian, C. E. (2005), «Conversion», dans L. Jones, Encyclopedia of Religion. Second Edition, Detroit, Macmillan, p. 19691974.

Salamito, J.-M. (1995), "La christianisation et les nouvelles règles sociales", dans J.-M. Mayeur, C. et L. Pietri, A. Vauchez et M. Venard, Histoire du christianisme 2, Naissance d'une chrétienté (250-430), Paris, Desclée, p. 675-717.

SANDERs, J. T. (2002), "Conversion in Early Christianity », dans A. J. Blasi, J. Duhaime et P. A. Turcotte, Handbook of Early Christianity. Social Science Approaches, Walnut Creek, Altamira Press, p. 619-641.

Saxer, V. (2000), "Culte et liturgie", dans J.-M. Mayeur,C. et L. Pietri, A. Vauchez et M. Venard, Histoire du christianisme 1, Le Nouveau Peuple (des origines jusqu'à 250), Paris, Desclée, p. 437-489. 
Steeman, T. M. (1975), "Church, Sect, Mysticism, Denomination: Periodological Aspects of Troeltsch's Types ", Sociological Analysis, 36, p. 181-204.

Stroumsa, G. (2005), La fin du sacrifice. Les mutations religieuses de l'Antiquité tardive, Paris, Odile Jacob.

Tardieu, M. (1997²), Le manichéisme, Paris, Presses Universitaires de France.

Torrance, A. (2013), Repentance in Late Antiquity. Eastern Asceticism and the Framing of the Christian Life c.400-650 CE, Oxford, Oxford University Press.

Troeltsch, E. (1919) [1912¹], «Die Soziallehren des christlichen Kirchen und Gruppen », dans Gesammelte Schriften 1, Tübingen, Mohr.

Vecoli, F. (2006), Lo Spirito soffia nel deserto. Carismi, discernimento e autorità nel monachesimo egiziano antico, Brescia, Morcelliana.

(2007), Il sole e il fango. Puro e impuro tra i Padri del deserto, Roma, Edizioni di Storia e Letteratura.

(2010), «Tassonomie spaziali nel monachesimo antico », Rivista di Storia del Cristianesimo, 7, p. 343-364

Veilleux, A. (1968), La liturgie dans le cénobitisme pachômien au quatrième siècle, Roma, Pontificium Institutum S. Anselmi.

Veyne, P. (2007), Quand notre monde est devenu chrétien (312-394), Paris, Albin Michel. 


\section{Résumé}

La conversion est au départ un phénomène judéo-chrétien, donc culturellement très connoté. Et c'est précisément dans cette perspective limitée que se situe notre intervention. L'intérêt est celui de mieux comprendre - d'un point de vue historique - une phase clé de l'évolution de la notion de conversion dans l'histoire du christianisme ancien. Il s'agit ici de remonter aux éléments premiers qui ont forgé le sens du mot tel que nous l'utilisons aujourd'hui en Occident. On ne se propose pas de forger la définition ultime, correspondante à la chose même à sa naissance, mais plutôt de saisir la dimension évolutive - donc dynamique - de la conversion, à travers l'étude d'une période charnière de son développement: c'est ce que l'on nomme ici le tournant monastique (IV-V siècles). Nous voulons montrer comment le phénomène de la conversion s'est transformé de façon significative à partir du $\mathrm{IV}^{\mathrm{e}}$ siècle de notre ère.

\section{Abstract}

At its beginning, conversion was a Judaeo-Christian phenomenon, that is to say a culturally connoted one. And this article was written with precisely this perspective in mind, the aim being to develop a better historical understanding of a key phase in the evolution of the notion of conversion in the history of late Ancient Christianity. We intend to trace back the very first components that made up the current meaning of the word "conversion". We do not pretend to provide the ultimate definition of this notion, nor the version most closely resembling its definition at the time of its emergence. Our intent is rather to recognize the dynamic aspect of conversion by studying a central period of its development. We have named this stage the "monastic turn" (IV-V ${ }^{\text {th }} c$.). In short, our purpose is to prove how - in the $I^{\text {th }}$ century C.E. - conversion underwent a significant transformation by the agency of Christian monasticism. 